\title{
CYCLIC SULLIVAN-DE RHAM FORMS
}

\author{
CHRISTOPHER ALLDAY
}

\begin{abstract}
For a simplicial set $X$ the Sullivan-de Rham forms are defined to be the simplicial morphisms from $X$ to a simplicial rational commutative graded differential algebra (cgda) $\nabla$. However $\nabla$ is a cyclic cgda in a standard way. And so, when $X$ is a cyclic set, one has a cgda of cyclic morphisms from $X$ to $\nabla$. It is shown here that the homology of this cgda is naturally isomorphic to the rational cohomology of the orbit space of the geometric realization $|X|$ with its standard circle action. In addition, a cyclic cgda $\nabla C$ is introduced; and it is shown that the homology of the cgda of cyclic morphisms from $X$ to $\nabla C$ is naturally isomorphic to the rational equivariant (Borel construction) cohomology of $|X|$.
\end{abstract}

\section{INTRODUCTION}

Recall that a simplicial set, $X$, is a graded set, graded over that natural numbers, $X_{0}, X_{1}, \ldots$, such that, for each $n \geq 1$, there are boundary maps $d_{i}: X_{n} \longrightarrow X_{n-1}, 0 \leq i \leq n$, and, for each $n \geq 0$, there are degeneracy maps $s_{j}: X_{n} \longrightarrow X_{n+1}, 0 \leq j \leq n$; and there are various relations amongst the $d_{i}$ 's and $s_{j}$ 's. (See $[\mathrm{M}]$.) More generally, a simplicial object in a category $\mathscr{C}$, for example, a simplicial group, a simplicial algebra or a simplicial topological space, is defined just as for a simplicial set, except that each $X_{n}$ is required to be an object of $\mathscr{C}$, and all $d_{i}$ 's and $s_{j}$ 's are required to be morphisms of $\mathscr{C}$. A cyclic set is a simplicial set with some additional structure: for each $n \geq 0, X_{n}$ is acted on by the cyclic group of order $n+1$; and, if $t_{n}$ denotes the generator of the cyclic group acting on $X_{n}$, then there are additional relations amongst the $d_{i}$ 's and the $t_{n}$ 's and amongst the $s_{j}$ 's and the $t_{n}$ 's. (See [L], §6.1.) A cyclic object in a category $\mathscr{C}$ (a cyclic group, a cyclic algebra or a cyclic topological space, for example) is a simplicial object in $\mathscr{C}$ with the additional group actions and relations, with each $t_{n}$ required to be a morphism of $\mathscr{C}$.

Alternatively, there is a simplicial category $\Delta$, and a simplicial object in a category $\mathscr{C}$ can be viewed as a contravariant functor $\Delta \longrightarrow \mathscr{C}$ (or a covariant functor from the opposite category $\Delta^{o p}$ to $\mathscr{C}$ ). Similarly there is a cyclic category $\Delta C$, which has the same objects as $\Delta$ and some additional morphisms; and a cyclic object in $\mathscr{C}$ is a contravariant functor $\Delta C \longrightarrow \mathscr{C}$ (or a covariant functor $\Delta C^{o p} \longrightarrow \mathscr{C}$ ). (Again see [L], §6.1.)

If $X$ is a simplicial set, then the commutative graded differential algebra (cgda) of Sullivan-de Rham forms on $X, A^{*}(X)$, is defined to be the cgda of

Received by the editors September 14, 1994.

1991 Mathematics Subject Classification. Primary 57S10; Secondary 55N91, 55P62, 18 G60. 
all simplicial maps of $X$ into a simplicial rational cgda $\nabla^{*}$. (See, e.g., [S], $[\mathrm{B}, \mathrm{G}],[\mathrm{H}]$. The precise definition will be reviewed below.) The homology of $A^{*}(X)$ is naturally isomorphic to the rational cohomology of $X$ or its geometric realization $|X|$. Now $\nabla^{*}$ has an obvious structure as a cyclic cgda. Thus, if $X$ is a cyclic set, then one can consider the cgda $A_{c y}^{*}(X)$ of all cyclic maps of $X$ into $\nabla^{*}$. One purpose of this paper is to show that the homology of $A_{c y}^{*}(X)$ is naturally isomorphic to the rational cohomology of $|X| / G$, where $G=S^{1}$, the circle group, acting on $|X|$ in the usual way (to be reviewed in $\S 2$ below). In addition we define another cyclic rational cgda, which we denote $\nabla C^{*}$, and we define $A_{G}^{*}(X)$ to be the cgda of all cyclic maps of $X$ into $\nabla C^{*}$. The other purpose of this paper is to show that the homology of $A_{G}^{*}(X)$ is naturally isomorphic to $H_{G}^{*}(|X| ; \mathbb{Q})$, the rational equivariant (Borel construction) cohomology of $|X|$.

To define $\nabla^{*}$ precisely one begins with the free rational cgda, $E_{n}$, say, generated by indeterminates $t_{n 0}, \ldots, t_{n n}$ of degree zero and their differentials $d t_{n 0}, \ldots, d t_{n n}$ of degree one. Then $\nabla_{n}^{*}:=E_{n} / J_{n}$ where $J_{n}$ is the ideal generated by $1-\sum_{j=0}^{n} t_{n j}$ and $\sum_{j=0}^{n} d t_{n j}$. The vector space of $q$-forms of simplicial dimension $n$ is denoted $\nabla_{n}^{q}$; and the simplicial vector space of $q$-forms is denoted $\nabla^{q}$. (See, e.g., [B, G] for details of the simplicial structure. In [B, G] $\nabla_{n}^{q}$ is denoted $\left.\nabla(n, q).\right)$

1.1. Definitions. The cyclic operator $t_{n}: \nabla_{n}^{*} \longrightarrow \nabla_{n}^{*}$ is induced by the cyclic permutation $\left(t_{n 0}, \ldots, t_{n n}\right) \mapsto\left(t_{n 1}, \ldots, t_{n n}, t_{n 0}\right)$. (Cf. [L], 7.1.3.)

If $X$ is a cyclic set, then let $A_{c y}^{*}(X)=\operatorname{Mor}_{\Delta C o p}\left(X, \nabla^{*}\right)$ and $A_{c y}^{q}(X)=$ $\operatorname{Mor}_{\Delta C^{o p}}\left(X, \nabla^{q}\right)$. Call $A_{c y}^{*}(X)$, resp. $A_{c y}^{q}(X)$, the cgda, resp. vector space, of rational cyclic Sullivan-de Rham forms, resp. $q$-forms, on $X$.

Now, if $X$ is a cyclic set, and $|X|$ is its geometric realization, then $G=S^{1}$ acts on $|X|$ in a standard way (see, e.g., [L], 7.1, to be reviewed in $\S 2$ below). One purpose of this paper is to prove the following theorem.

1.2. Theorem. Given a cyclic set $X$ there is a natural isomorphism of rational commutative graded algebras

$$
H\left(A_{c y}^{*}(X)\right) \cong H^{*}(|X| / G ; \mathbb{Q}) .
$$

In $\S 5$ below we define the cyclic rational cgda $\nabla C^{*}$. Then, for a cyclic set $X$, we define $A_{G}^{*}(X)=\operatorname{Mor}_{\Delta C^{o p}}\left(X, \nabla C^{*}\right)$. The second result of this paper is the following.

1.3. Theorem. Given a cyclic set $X$ there is a natural isomorphism of rational commutative graded algebras

$$
H\left(A_{G}^{*}(X)\right) \cong H_{G}^{*}(|X| ; \mathbb{Q}) .
$$

Both proofs are basically cyclic versions of the proof in the simplicial case to be found in $[B, G], \S \S 14$ and 3 . They make essential use of some constructions to be found in [B, H, M] and [Sp]: and I would like to thank Jan Spaliński for his very timely visit to Hawaii and for his very helpful paper.

In $\S 2$ below we review some basic facts concerning cyclic sets. In $\S 3$ we prove the additive part of Theorem 1.2. And in $\S 4$ we deal with the multiplicative part. Theorem 1.3 is proven in $\S 5$. 


\section{REVIEW OF CYCLIC SETS.}

As far as possible we shall follow the notation used in [L]. However we shall frequently write $\Lambda[n]$ instead of $F \Delta[n]$, where $F$ is the left adjoint of the forgetful functor $\Delta C^{o p} \longrightarrow \Delta^{o p} \quad([\mathrm{~L}], 7.1 .5)$. And $t_{n}$ will denote the cyclic operator without sign ([L], 6.1.2).

Now let $X$ be a cyclic set. And let $|X|=\coprod_{n \geq 0} X_{n} \times \Delta_{n} / \sim$ be its geometric realization defined, just as in $[\mathrm{M}], \S 14$, using only the simplicial structure. Let $[x, u] \in|X|$ be the equivalence class of $(x, u) \in X_{n} \times \Delta_{n}$, where $x$ is nondegenerate and $u=\left(u_{0}, \ldots, u_{n}\right) \in \Delta_{n}$ is interior. The canonical circle action on $|X|$ is given by

$$
e^{2 \pi i v}[x, u]=\left[t_{n+1}^{n+1-j} s_{j} x, \tau_{n+1}^{j+1}\left(w_{0}, \ldots, w_{n+1}\right)\right]
$$

where $0 \leq v<1, \tau_{n+1}$ is the cocyclic operator, i.e. $\tau_{n+1}\left(w_{0}, \ldots, w_{n+1}\right)=$ $\left(w_{1}, \ldots, w_{n+1}, w_{0}\right)$, and $\left(w_{0}, \ldots, w_{n+1}\right)=\left(u_{0}, \ldots, u_{j-1}, 1-v-u^{j-1}, u^{j}-\right.$ $\left.(1-v), u_{j+1}, \ldots, u_{n}\right)$, where $u^{j}=u_{0}+\cdots+u_{j}, u^{-1}=0$ and $j$ is such that $u^{j-1}<1-v \leq u^{j}$. (See [L], 7.1, and [M], proof of Theorem 14.3.)

2.1. Definition. For a cyclic set $X$ let

$$
X_{0}^{f}=\left\{x \in X_{0} ; t_{1} s_{0} x=s_{0} x\right\} .
$$

And let $X^{f}$ be the cyclic subset of $X$ generated by $X_{0}^{f}$. (For any $y \in X_{n}^{f}$, $y=s_{0}^{n} x$ for some $x \in X_{0}^{f}$, and $t_{n} y=y$.)

Clearly $\left|X^{f}\right|=|X|^{G}$. (For a cyclic set $X$, the fixed point set is always discrete.)

2.2. Remark. It is well-known that $|X| \approx \coprod_{n \geq 0} X_{n} \times \Lambda_{n} / \sim$ where $\Lambda_{n}=|\Lambda[n]|$ and, now, the equivalence relation uses all cyclic operators (i.e. all operators from $\Delta C)\left([\mathrm{D}, \mathrm{H}, \mathrm{K}]\right.$, Proposition 2.8). However, $\Delta_{*}$ is also a cocyclic space with $\tau_{n}$ as above. So one may form $|X|_{o r b}:=\coprod_{n \geq 0} X_{n} \times \Delta_{n} / \sim$ using all operators from $\Delta C$. It is easy to see that there is a canonical homeomorphism $|X|_{\text {orb }} \approx$ $|X| / G$.

Given a cyclic set $X$, the group $\mathbb{Z} /(n+1)$ generated by $t_{n}$ acts on $X_{n}$; and so each $x \in X_{n}$ has an isotropy subgroup equal to a cyclic group $K_{r}$ of order $r$ for some $r$ dividing $n+1$. The proofs of the following technical lemma and Corollary 2.4 will be given in the appendix.

2.3. Lemma. Let $Y, Z$ be cyclic sets. Let $x \in Y_{n}$. Suppose that $t_{n}^{q} x$ is nondegenerate for all $q(0 \leq q \leq n)$, and that $x \notin Y_{0}^{f}$. Suppose that $x$ has isotropy subgroup $K_{r}$. Finally suppose that $t_{n+k}^{m_{1}} s_{i_{1}} \ldots s_{i_{k}} x=t_{n+k}^{m_{2}} s_{j_{1}} \ldots s_{j_{k}} x$ in $Y_{n+k}$ for some $k \geq 0$.

Then $t_{n+k}^{m_{1}} s_{i_{1}} \ldots s_{i_{k}} z=t_{n+k}^{m_{2}} s_{j_{1}} \ldots s_{j_{k}} z$ for any $z \in Z_{n}$ if the isotropy subgroup of $z$ contains $K_{r}$.

Recall that if $r \mid n+1$, then there is a cyclic action of $K_{r}$ on $\Lambda$ [n]. (See [Sp], 3.5. In the notation of [L], 7.1, the action of the generator of $K_{n+1}$ on $\Lambda[n]=$ $F \Delta[n]$ is the map $\Lambda[n] \longrightarrow \Lambda[n]$ corresponding to the point $\left(t_{n}, l_{n}\right)$ : i.e. $\left(1, l_{n}\right) \mapsto\left(t_{n}, l_{n}\right)$.) Let $\dot{\Lambda}[n]=F \dot{\Delta}[n]$ be the usual cyclic subset of boundaries. The following corollaries follow from Lemma 2.3. 
2.4. Corollary. Let $Y$ be a cyclic set and $X \subseteq Y$ a cyclic subset. Let $x \in$ $Y_{n}-X_{n}$. Suppose that $t_{n}^{k} x$ is non-degenerate for $0 \leq k \leq n$, that $x \notin Y_{0}^{f}$ and that $d_{i} x \in X_{n-1}$ for $0 \leq i \leq n$. Suppose that $x$ has isotropy subgroup $K_{r}$.

Then the following diagram is a push-out.

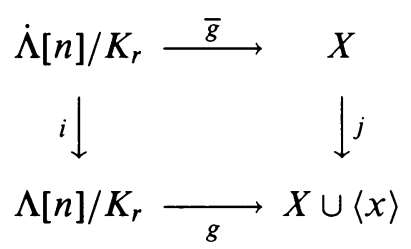

where $X \cup\langle x\rangle$ is the cyclic subset of $Y$ generated by $X$ and $x$, the vertical maps are the inclusions, and $g$ is induced by $\left(1, l_{n}\right) \mapsto x$.

2.5. Corollary. Let $X$ be a cyclic set. Let $X(n)$ be the $n$-skeleton of $X$, i.e. the cyclic subset generated by $\bigcup_{j=0}^{n} X_{j}$. Then $X$ is the direct limit of the sequence $X(-1):=X^{f} \subseteq X(0) \subseteq X(1) \subseteq \cdots \subseteq X(n-1) \subseteq X(n) \ldots$, and each $X(n-1) \subseteq X(n)$, for $n \geq 0$, is a push-out

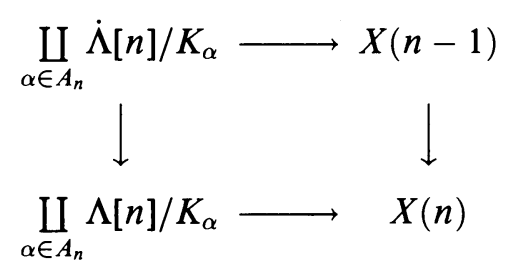

where $A_{n}$ is the set of orbits of simplicies $x \in X_{n}$ such that $t_{n}^{k} x$ is nondegenerate for $0 \leq k \leq n$, and $K_{\alpha}$ is the isotropy subgroup of the orbit $\alpha$. $\left(A_{0}=X_{0}-X_{0}^{f}\right.$.)

Since geometric realization is a left adjoint, and so commutes with colimits, one also gets the following.

2.6. Corollary. If $X$ is a cyclic set, then $|X|$ is a $G-C W$-complex (where $G=$ $\left.S^{1}\right)$.

The next lemma is also useful.

2.7. Lemma. Let $Z$ be an acyclic cyclic rational vector space. (I.e., $Z$ is a cyclic rational vector space, and $Z \longrightarrow 0$ is a homotopy equivalence of simplicial abelian groups.) Then the dotted arrow exists in any commutative diagram of the form

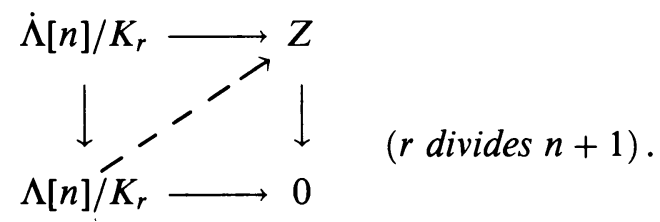


Proof. By $[\mathrm{D}, \mathrm{H}, \mathrm{K}], \lambda$ exists in the diagram

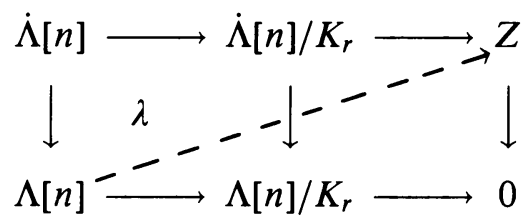

since the vertical map on the right is an acyclic fibration and the vertical map on the left is a cofibration.

Suppose that $\lambda\left(1, l_{n}\right)=\omega$. (Here, as above, $l_{n} \in \Delta[n]$ is the generator, and we are thinking of $\Lambda[n]$ as $F \Delta[n]$ as in [L], 7.1.) Now let $\theta=\frac{1}{r} \sum_{j=0}^{r-1} t_{n}^{j s} \omega$, where $r s=n+1$. Define $\mu: \Lambda[n] \longrightarrow Z$ by $\mu\left(1, l_{n}\right)=\theta$. A straightforward check shows that $d_{i} \theta=d_{i} \omega$ for $0 \leq i \leq n$. So $\mu$ also makes the above diagram commute. And $\mu$ factors through $\Lambda[n] / K_{r}$.

\section{The ADDITIVe PART OF TheOREM 1.2.}

Here we verify that a cyclic version of $[B, G], \S 14$ is valid. First, however, recall the Connes cochain complex $S_{\lambda}^{*}(X)$ of a cyclic set $X$ with rational coefficients. A cyclic cochain $\varphi \in S_{\lambda}^{n}(X)$ is an ordinary cochain $\varphi: X_{n} \longrightarrow \mathbb{Q}$ such that $\varphi\left(t_{n} x\right)=(-1)^{n} \varphi(x)$ for all $x \in X_{n}$ ([L], 2.5.9). Then (see [J] or, e.g., [L], 7.2.3)

$$
H\left(S_{\lambda}^{*}(X)\right) \cong H_{G}^{*}(|X| ; \mathbb{Q}),
$$

the equivariant (Borel construction) cohomology.

Recall, too, the map $\rho: A^{*}(X) \longrightarrow S^{*}(X)$ from the Sullivan-de Rham cgda of a simplicial set $X$ to the rational cochain complex of $X$ ([B, G], p. 7). For $\varphi \in A^{n}(X)$ and $x \in X_{n}, \rho(\varphi)(x)=\int \varphi(x)$, where the integration is over $\Delta_{n}^{\prime}:=\left\{\left(v_{1}, \ldots, v_{n}\right) \in \mathbb{R}^{n} ; \sum_{i=1}^{n} v_{i} \leq 1\right.$ and $v_{i} \geq 0$ for $\left.1 \leq i \leq n\right\}$.

3.1. Lemma. For any cyclic set $X$, the restriction of $\rho$ to $A_{c y}^{*}(X)$ maps into $S_{\lambda}^{*}(X)$ : i.e. one has

$$
\rho: A_{c y}^{*}(X) \longrightarrow S_{\lambda}^{*}(X)
$$

Proof. We must show that $(-1)^{n} \rho(\varphi)(x)=\rho(\varphi)\left(t_{n} x\right)$ for $\varphi \in A_{c y}^{n}(X)$ and $x \in X_{n}$. Let $\varphi(x)=f\left(t_{n 1}, \ldots, t_{n n}\right) d t_{n 1} \ldots d t_{n n}$. Then

$$
\varphi\left(t_{n} x\right)=t_{n} \varphi(x)=f\left(t_{n 2}, \ldots, t_{n n}, 1-t\right) d t_{n 2} \ldots d t_{n n} d(1-t),
$$

where $t=\sum_{i=1}^{n} t_{n i}$. So $\varphi\left(t_{n} x\right)=(-1)^{n} f\left(t_{n 2}, \ldots, t_{n n}, 1-t\right) d t_{n 1} \ldots d t_{n n}$. Now the change of variable $v_{1}=t_{n 2}, \ldots, v_{n-1}=t_{n n}, v_{n}=1-t$ shows that $\int \varphi\left(t_{n} x\right)=(-1)^{n} \int \varphi(x)$.

3.2. Notation. For a cyclic set $X$ let

$$
\widetilde{A}_{c y}^{*}(X)=A_{c y}^{*}\left(X, X^{f}\right)=\operatorname{ker}\left[A_{c y}^{*}(X) \longrightarrow A_{c y}^{*}\left(X^{f}\right)\right]
$$

and

$$
\widetilde{S}_{\lambda}^{*}(X)=S_{\lambda}^{*}\left(X, X^{f}\right)=\operatorname{ker}\left[S_{\lambda}^{*}(X) \longrightarrow S_{\lambda}^{*}\left(X^{f}\right)\right]
$$


Note that $\rho$ induces $\tilde{\rho}: \widetilde{A}_{c y}^{*}(X) \longrightarrow \widetilde{S}_{\lambda}^{*}(X)$. And, by $[\mathrm{J}], H \widetilde{S}_{\lambda}^{*}(X)$ is naturally isomorphic to $H_{G}^{*}\left(|X|,|X|^{G} ; \mathbb{Q}\right)$.

If $Y$ is a cyclic set and $X \subseteq Y$ is a cyclic subset, then $Y$ is the direct limit of the sequence

$$
X \subseteq X \cup Y^{f} \subseteq X \cup Y(0) \subseteq \cdots \subseteq X \cup Y(n-1) \subseteq X \cup Y(n) \subseteq \ldots,
$$

where $Y(n)$ is the cyclic $n$-skeleton of $Y$ (as in Corollary 2.5). Thus the next lemma follows easily from Corollary 2.5 and Lemma 2.7.

3.3. Lemma. Let $Y$ be a cyclic set and $X \subseteq Y$ a cyclic subset. Then the restriction homomorphism $A_{c y}^{*}(Y) \longrightarrow A_{c y}^{*}(X)$ is surjective.

Indeed, since the extension to $Y^{f}$ can be arbitrary, the restriction homomorphism $\widetilde{A}_{c y}^{*}(Y) \longrightarrow \widetilde{A}_{c y}^{*}(X)$ is surjective also.

The next lemma is an easy variant of the corresponding simplicial result (see, e.g., [B, G], p. 82).

3.4. Lemma. Let $X: J \longrightarrow$ cyclic sets be a functor from a small category $J=\{j\}$ to the category of cyclic sets. Suppose that the map $\underset{\lim X(j)}{\longrightarrow} \longrightarrow$ $(\underline{\lim } X(j))^{f}$ is surjective. Then $\widetilde{A}_{c y}^{*}(\underline{\lim } X(j)) \cong \underline{\lim } \widetilde{A}_{c y}^{*}(X(j))$. (Here $\underset{\lim }{\longrightarrow}$ is colimit and $\underline{\lim }$ is limit.) Similarly $\widetilde{S}_{\lambda}^{*}(\underline{\lim } X(j)) \cong \underline{\lim } \widetilde{S}_{\lambda}^{*}(X(j))$.

It is also easy to verify the following.

\subsection{Lemma. Let}

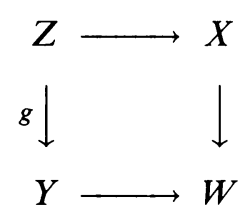

be a push-out of cyclic sets.

Let $\bar{W}$ be the push-out obtained by replacing $X, Y$ and $Z$ by $X^{f}, Y^{f}$ and $Z^{f}$ respectively. Let $h: \bar{W} \longrightarrow W^{f}$ be the standard map.

If $g$ is injective, then $h$ is surjective. If, in addition, $Z^{f}=Y^{f}=\varnothing$, then $h$ is bijective.

3.6. Lemma. For any $n \geq 0$ and $r$ dividing $n+1$, the map

$$
\tilde{\rho}: \tilde{A}_{c y}^{*}\left(\Lambda[n] / K_{r}\right) \longrightarrow \widetilde{S}_{\lambda}^{*}\left(\Lambda[n] / K_{r}\right)
$$

induces an isomorphism in homology.

Proof. It is easy to see that $\left(\Lambda[n] / K_{r}\right)^{f}=\varnothing$. So we are concerned with $\rho$ : $A_{c y}^{*}\left(\Lambda[n] / K_{r}\right) \longrightarrow S_{\lambda}^{*}\left(\Lambda[n] / K_{r}\right)$.

Now with the notation and results of [Sp] we have the following (where $r s=n+1)$.

$$
\begin{aligned}
A_{c y}^{*}\left(\Lambda[n] / K_{r}\right) & =\operatorname{Mor}_{\Delta C^{o p}}\left(\Psi_{r} \Delta[s-1], \nabla^{*}\right) \\
& =\operatorname{Mor}_{\Delta^{o p}}\left(\Delta[s-1], \Phi_{r}\left(\nabla^{*}\right)\right) \\
& \cong \Phi_{r}\left(\nabla^{*}\right)_{s-1}=\left(\nabla_{n}^{*}\right)^{K_{r}}
\end{aligned}
$$


Since $H\left(\nabla_{n}^{*}\right)=\mathbb{Q}, H\left(\left(\nabla_{n}^{*}\right)^{K_{r}}\right)=\mathbb{Q}$ by averaging.

On the other hand,

$$
\begin{aligned}
H\left(S_{\lambda}^{*}\left(\Lambda[n] / K_{r}\right)\right) & \cong H_{G}^{*}\left(\left|\Lambda[n] / K_{r}\right| ; \mathbb{Q}\right) \\
& \cong H_{G}^{*}\left(|\Lambda[n]| / K_{r} ; \mathbb{Q}\right) \\
& \cong H_{G}^{*}(|\Lambda[n]| ; \mathbb{Q}) \text { since } K_{r} \text { is finite } \\
& \cong \mathbb{Q} .
\end{aligned}
$$

We are now in a position to prove the following, which essentially gives the additive part of Theorem 1.2. (See proof of 4.8.)

3.7. Proposition. Let $X$ be a cyclic set. Then $\tilde{\rho}: \widetilde{A}_{c y}^{*}(X) \longrightarrow \widetilde{S}_{\lambda}^{*}(X)$ induces an isomorphism $\widetilde{\rho}^{*}: H\left(A_{c y}^{*}\left(X, X^{f}\right)\right) \longrightarrow H\left(S_{\lambda}^{*}\left(X, X^{f}\right)\right)$.

Proof. We can now mimic the proof in $[\mathrm{B}, \mathrm{G}], 14.5$.

Step 1 holds for the standard cyclic sets $\Lambda[n] / K_{r}$ by Lemma 3.6. Step 2 holds by Step 1 and Lemma 3.4. Step 3 follows from Step 2, induction, Corollary 2.5, Lemma 3.4 and, in order to get the required version of $[B, G]$, Lemma 14.1, Lemma 3.3. Finally Step 4 follows from Step 3, Lemma 3.4 and Lemma 3.3, which permits the required version of $[B, G]$, Lemma 14.4 .

\section{THE MULTIPLICATIVE PART OF THEOREM 1.2.}

Unfortunately, although the methods of $[B, G], \S 14$, seem to work best for the additive part, acyclic model arguments seem to be needed for the multiplicative part. We shall follow as closely as possible the notation of $[B, G], \S 2$.

4.1. Definitions. Let $K$ be a contravariant functor from the category of cyclic sets to the category of $R$-modules, where $R$ is a commutative ring with identity. For a cyclic set $X$ let $\widehat{K}(X)=\prod_{n \geq 0} \widetilde{\prod_{x \in X_{n}}}\{K(\Lambda[n]), x\}$, where $\widetilde{\Pi}$ indicates the submodule of the product consisting of elements $\left\{m_{x}, x\right\}$ where (for $x \in X_{n}$ ) $m_{t_{n} x}=\gamma_{n}^{*} m_{x}, \gamma_{n}: \Lambda[n] \longrightarrow \Lambda[n]$ is the cyclic map induced by $\gamma_{n}\left(1, l_{n}\right)=$ $\left(t_{n}, l_{n}\right)$, and $\gamma_{n}^{*}=K\left(\gamma_{n}\right)$.

Define $\Phi: K \longrightarrow \widehat{K}$ by $\Phi(X)(u)=\{K(\tilde{x})(u), x\}$, for a cyclic set $X$ and $u \in K(X)$, where $\tilde{x}: \Lambda[n] \longrightarrow X$ is the standard map corresponding to $x \in X_{n}$ (i.e. $\tilde{x}\left(1, t_{n}\right)=x$ ). It follows that $\Phi(X)$ maps $K(X)$ to $\widehat{K}(X)$ since $\widetilde{t_{n} x}=\widetilde{x} \gamma_{n}$.

The functor $K$ is said to be corepresentable (with respect to the models $\Lambda[n])$ if there is a natural transformation $\Psi: \widehat{K} \longrightarrow K$ such that $\Psi \Phi=1$.

4.2. Lemma. The functor $S_{\lambda}^{*}$ is corepresentable (w.r.t. the models $\Lambda[n]$ ).

Proof. Given a cyclic set $X,\left\{m_{x}, x\right\} \in \widehat{S}_{\lambda}^{n}(X)$ and $y \in X_{n}$, let

$$
\Psi(X)\left(\left\{m_{x}, x\right\}\right)(y)=m_{y}\left(1, l_{n}\right) .
$$

Since $m_{t_{n} y}=\gamma_{n}^{*} m_{y}$, it follows that $\Psi(X)\left(\left\{m_{x}, x\right\}\right)$ is a cyclic cochain. And it is immediate that $\Psi \Phi=1$. 
4.3. Lemma. The functors $A_{c y}^{*}, S_{\lambda}^{*}, A_{c y}^{*} \otimes A_{c y}^{*}$ and $S_{\lambda}^{*} \otimes S_{\lambda}^{*}$ are acyclic with respect to the models $\Lambda[n]$ in the sense of $[\mathrm{B}, \mathrm{G}], p .9$.

Proof. This follows exactly as in $[\mathrm{B}, \mathrm{G}]$ since $A_{c y}^{*}(\Lambda[n]) \cong A^{*}(\Delta[n])$ and $S_{\lambda}^{*}(\Lambda[n]) \cong S^{*}(\Delta[n])$.

Suppose that $K^{*}$ is a functor (such as those of Lemma 4.3) which is acyclic with respect to the models $\Lambda[n]$ in the sense of $[\mathrm{B}, \mathrm{G}], \mathrm{p}$. 9. So, for fixed $n$ and any $p$, we have a homotopy $h: K^{p}(\Lambda[n]) \longrightarrow K^{p-1}(\Lambda[n])$ such that $h D+D h=$ $1-\eta \epsilon$, where $D$ is the differential in $K^{*}(\Lambda[n])$, and $\eta: \mathbb{Q} \longrightarrow K^{\circ}(\Lambda[n])$ and $\epsilon: K^{*}(\Lambda[n]) \longrightarrow \mathbb{Q}$ are the unit and augmentation (so that $\epsilon \eta=1$ ). In order to apply the obvious cyclic analogues of [B, G], Lemma 2.3 and Proposition 2.4 , it is necessary that $h \gamma_{n}^{*}=\gamma_{n}^{*} h$. But this is easily done by starting with any $h$ and averaging to obtain $\tilde{h}:=\frac{1}{n+1} \sum_{j=0}^{n}\left(\gamma_{n}^{*}\right)^{j} h\left(\gamma_{n}^{*}\right)^{-j}$. One must also average $\epsilon$ by putting $\tilde{\epsilon}=\frac{1}{n+1} \sum_{j=0}^{n} \epsilon\left(\gamma_{n}^{*}\right)^{-j}$. One then has $\tilde{h} D+D \tilde{h}=1-\eta \widetilde{\epsilon}$, provided that the image of $\eta$ is in the fixed part of $K^{\circ}(\Lambda[n])$ : and that is the case for all functors considered here.

Thus one has available the cyclic analogues of [B, G], Lemma 2.3 and Proposition 2.4. Hence one has the following.

\subsection{Corollary. There is a natural chain map}

$$
\mu_{\lambda}: S_{\lambda}^{*} \otimes S_{\lambda}^{*} \longrightarrow S_{\lambda}^{*} \text {. }
$$

which is homotopy associative, homotopy commutative, has a homotopy unit and is unique up to natural chain homotopy.

By naturality $\mu_{\lambda}$ induces $\widetilde{\mu}_{\lambda}: \widetilde{S}_{\lambda}^{*} \otimes S_{\lambda}^{*} \longrightarrow \widetilde{S}_{\lambda}^{*}$ and $\widetilde{\tilde{\mu}}_{\lambda}: \widetilde{S}_{\lambda}^{*} \otimes \widetilde{S}_{\lambda}^{*} \longrightarrow \widetilde{S}_{\lambda}^{*}$. Let $\mu_{A}: A_{c y}^{*} \otimes A_{c y}^{*} \longrightarrow A_{c y}^{*}$ be the usual multiplication of forms; and let $\tilde{\mu}_{A}$ and $\widetilde{\tilde{\mu}}_{A}$ be the corresponding restrictions. Then [B, G], Lemma 2.3 and Proposition 2.4 also give the following.

4.5. Proposition. There are natural chain homotopies $\rho \mu_{A} \simeq \mu_{\lambda}(\rho \otimes \rho), \tilde{\rho} \tilde{\mu}_{A} \simeq$ $\tilde{\mu}_{\lambda}(\tilde{\rho} \otimes \rho)$ and $\tilde{\rho} \tilde{\tilde{\mu}}_{A} \simeq \tilde{\tilde{\mu}}_{\lambda}(\tilde{\rho} \otimes \tilde{\rho})$. Furthermore, if $\mu: S^{*} \otimes S^{*} \longrightarrow S^{*}$ is the standard cup product (as in [M], §30) and if $i: S_{\lambda}^{*} \longrightarrow S^{*}$ is the inclusion, then there is a natural chain homotopy $i \mu_{\lambda} \simeq \mu(i \otimes i)$.

Proof. The first natural chain homotopy follows from [B, G], Lemma 2.3 and Proposition 2.4. (See also [B, G], Proposition 3.3.) The second and third follow from the first by naturality. The last follows since $S^{*}$ is corepresentable with respect to the models $\Lambda[n]$ if in the definition of $\widehat{S}^{*}$ one uses the product $\Pi$ instead of the limit $\widetilde{\Pi}$. (And one is viewing $S^{*}$ as a functor on cyclic sets not simplicial sets.)

4.6. Corollary. For any cyclic set $X, \rho$ induces $\mathbb{Q}$-algebra homomorphisms $\rho^{*}: H\left(A_{c y}^{*}(X)\right) \longrightarrow H\left(S_{\lambda}^{*}(X)\right)$ and $\widetilde{\rho}^{*}: H\left(\widetilde{A}_{c y}^{*}(X)\right) \longrightarrow H\left(\widetilde{S}_{\lambda}^{*}(X)\right)$, the second being an isomorphism.

Remark. The functors $\widetilde{S}_{\lambda}^{*}, A_{c y}^{*}$ and $\widetilde{A}_{c y}^{*}$ cannot be corepresentable (w.r.t. the models $\Lambda[n])$. Otherwise one would get chain equivalences which are clearly impossible. The fact that $A_{c y}^{*}$ is not corepresentable w.r.t. $\Lambda[n]$ whereas $A^{*}$ 
is corepresentable w.r.t. $\Delta[n]$ ([B, G], Proposition 2.5) corresponds to the facts that $\mathbb{Q} \longrightarrow \nabla^{0} \longrightarrow \nabla^{1} \longrightarrow \cdots$ is an injective resolution of $\mathbb{Q}$ in the category of simplicial rational vector spaces but not in the category of cyclic rational vector spaces. The former fact gives a quick proof that, for any simplicial set $X, H\left(A^{*}(X)\right) \cong H^{*}(X ; \mathbb{Q})$. (See, e.g., [L], 6.2.)

We now restate and prove Theorem 1.2.

4.8. Theorem. For cyclic sets $X$ there is a natural isomorphism of $\mathbb{Q}$-algebras

$$
H\left(A_{c y}^{*}(X)\right) \stackrel{\sim}{\longrightarrow} H^{*}(|X| / G ; \mathbb{Q}) .
$$

(Here, as usual, $G=S^{1}$.)

Proof. From [J] one has natural isomorphisms $H\left(S_{\lambda}^{*}(X)\right) \cong H_{G}^{*}(|X| ; \mathbb{Q})$ and $H\left(\widetilde{S}_{\lambda}^{*}(X)\right) \cong H_{G}^{*}\left(|X|,|X|^{G} ; \mathbb{Q}\right)$. In addition there is a natural isomorphism $H_{G}^{*}\left(|X|,|X|^{G} ; \mathbb{Q}\right) \longrightarrow H^{*}\left(|X| / G,|X|^{G} ; \mathbb{Q}\right)$. (See, e.g., [A, P], Proposition (3.10.9), and Corollary 2.6 above.)

Thus, for $n \geq 2$, one has the sequence of isomorphisms $H^{n}\left(A_{c y}^{*}(X)\right) \longrightarrow$ $H^{n}\left(\widetilde{A}_{c y}^{*}(X)\right) \longrightarrow H^{n}\left(\tilde{S}_{\lambda}^{*}(X)\right) \longrightarrow H_{G}^{n}\left(|X|,|X|^{G} ; \mathbb{Q}\right) \longrightarrow H^{n}\left(|X| / G,|X|^{G} ; \mathbb{Q}\right)$ $\longrightarrow H^{n}(|X| / G ; \mathbb{Q})$, using the fact that $|X|^{G}$ is discrete.

The cases where $n=0$ or 1 are straightforward. The multiplicativity also follows easily thanks to Proposition 4.5.

\section{Proof of Theorem 1.3.}

In this section we define $\nabla C^{*}$ and $A_{G}^{*}$, and prove Theorem 1.3.

5.1. Definitions. (1) Let $R_{n}$ be the rational cgda $\mathbb{Q}\left[u_{n}\right] \otimes \Lambda\left(v_{n}\right)$, where $\operatorname{deg}\left(v_{n}\right)$ $=1, \operatorname{deg}\left(u_{n}\right)=2$ and $d v_{n}=u_{n}$.

(2) Let $R$ be the simplicial cgda which is $R_{n}$ in simplicial dimension $n$, and in which the simplicial operators are defined by requiring that $u_{n}=s_{0}^{n} u_{0}$ and $v_{n}=s_{0}^{n} v_{0}$, the $n$-fold degeneracies.

(3) The cyclic rational cgda $\nabla C^{*}$ is defined as follows. Let $\nabla C_{n}^{*}=R_{n} \otimes \nabla_{n}^{*}$. The simplicial operators are the tensors of those on $R_{n}$ with those on $\nabla_{n}^{*}$. (E.g., for $\varphi \in \nabla_{n}^{*}$ and $0 \leq i \leq n, d_{i}\left(u_{n} \otimes \varphi\right)=u_{n-1} \otimes d_{i} \varphi$.) The cyclic group operators are given as before on $\nabla^{*}$ (Definitions 1.1) and by requiring that $t_{n} u_{n}=u_{n}$ and $t_{n} v_{n}=v_{n}-d t_{n 0}$, for all $n \geq 0$.

It is easy to check that $\nabla C^{*}$ is a cyclic rational cgda. (E.g., $s_{0} t_{n} v_{n}=v_{n+1}-$ $d t_{n+10}-d t_{n+11}=t_{n+1}^{2} v_{n+1}=t_{n+1}^{2} s_{n} v_{n}$.)

(4) If $X$ is a cyclic set, then let

$$
A_{G}^{*}(X)=\operatorname{Mor}_{\Delta C^{o p}}\left(X, \nabla C^{*}\right) .
$$

It is also easy to check the following.

5.2. Lemma. For each degree $q$, the cyclic rational vector space $\nabla C^{q}$ is acyclic in the sense of Lemma 2.7.

Hence (cf. Lemma 3.3) one has the next corollary.

5.3. Corollary. If $Y$ is a cyclic set and $X \subseteq Y$ is a cyclic subset, then the restriction homomorphism $A_{G}^{*}(Y) \longrightarrow A_{G}^{*}(X)$ is surjective.

Since $\nabla C^{\circ}=\mathbb{Q} \otimes \nabla^{\circ} \cong \nabla^{\circ}$, one has that $A_{G}^{\circ}=A_{c y}^{\circ}$. Thus one has $\eta: \mathbb{Q} \longrightarrow$ $A_{G}^{\circ}(\Lambda[n])$ as before. (See Lemma 4.3 and the comments below it.) 
5.4. Lemma. The functors $A_{G}^{*}$ and $A_{G}^{*} \otimes A_{G}^{*}$ are acyclic with respect to the models $\Lambda[n]$.

Proof. One has that $A_{G}^{*}(\Lambda[n])=\nabla C_{n}^{*}=R_{n} \otimes \nabla_{n}$. One begins by defining $h$ on $R_{n}$ by $h(1)=0, h\left(u_{n}^{j}\right)=v_{n} u_{n}^{j-1}$ for $j \geq 1$ and $h\left(v_{n} u_{n}^{i}\right)=0$. And $h$ is defined on $\nabla_{n}$ in the usual way ([B, G], Proposition 1.3). One defines $\epsilon$ on $R_{n}$ by $\epsilon\left(v_{n}\right)=0, \epsilon\left(u_{n}\right)=0$. Then $h$ is defined on $R_{n} \otimes \nabla_{n}$ in the standard way (i.e., $h(x \otimes y)=\frac{1}{2}\left\{h(x) \otimes(y+\eta \epsilon(y))+(-1)^{m}(x+\eta \epsilon(x)) \otimes h(y)\right\}$, where $\operatorname{deg}(x)=m)$. Then one averages as in the comments below Lemma 4.3.

Everything is now in place to apply the cyclic analogues of $[B, G]$, Lemma 2.3 and Proposition 2.4, which give the following.

5.5. Proposition. There is a natural chain map $\rho_{G}: A_{G}^{*} \longrightarrow S_{\lambda}^{*}$ such that

(1) $\rho_{G} \eta=\eta: \mathbb{Q} \longrightarrow S_{\lambda}^{\circ}$;

(2) in degree $0, \rho_{G}=\rho: A_{G}^{\circ}=A_{c y}^{\circ} \longrightarrow S_{\lambda}^{\circ}$ (see Lemma 3.1);

(3) there is a natural chain homotopy $\rho_{G} i_{\nabla^{*}} \simeq \rho: A_{c y}^{*} \longrightarrow S_{\lambda}^{*}$, where $i_{\nabla^{*}}: A_{c y}^{*} \longrightarrow A_{G}^{*}$ is induced by the obvious inclusion $i_{\nabla}: \nabla^{*} \longrightarrow \nabla C^{*}$ of cyclic cgdas; and

(4) there is a natural chain homotopy

$$
\rho_{G} \mu_{G} \simeq \mu_{\lambda}\left(\rho_{G} \otimes \rho_{G}\right): A_{G}^{*} \otimes A_{G}^{*} \longrightarrow S_{\lambda}^{*},
$$

where $\mu_{G}$ is the multiplication on $A_{G}^{*}$ and $\mu_{\lambda}$ is a multiplication on $S_{\lambda}^{*}$ given by Corollary 4.4 .

Finally we are ready to restate and prove Theorem 1.3.

5.6. Theorem. For cyclic sets $X, \rho_{G}$ of Proposition 5.5 induces a natural isomorphism of rational commutative graded algebras

$$
\rho_{G}^{*}: H\left(A_{G}^{*}(X)\right) \stackrel{\sim}{\longrightarrow} H_{G}^{*}(|X| ; \mathbb{Q}) .
$$

Proof. As in the proof of Proposition 3.7, we mimic the proof in [B, G], 14.5. The multiplicative part of the theorem follows from Proposition 5.5(4).

Step 1 of $[\mathrm{B}, \mathrm{G}]$ follows for the cyclic sets $\Lambda[n] / K_{r}$, because, arguing as in Lemma 3.6, one has that

$$
A_{G}^{*}\left(\Lambda[n] / K_{r}\right) \cong\left(\nabla C_{n}^{*}\right)^{K_{r}}
$$

and, as before, the homology of the latter is $\mathbb{Q}$ concentrated in degree 0 . There is, however, the crucial question of what happens on the trivial cyclic set $\Delta[0]$. We shall postpone this to last.

The remaining steps of $[B, G], 14.5$ follow just as in the proof of Proposition 3.7, but using Corollary 5.3 instead of Lemma 3.3.

So, returning to $\Delta[0]$, one has that

$$
A_{G}^{*}(\Delta[0]) \cong\left(\nabla C^{*}\right)_{0}^{f}=\mathbb{Q}\left[u_{0}\right],
$$

the polynomial ring. And $S_{\lambda}^{*}(\Delta[0])=\mathbb{Q}[w]$, the polynomial ring on $w \in$ $S_{\lambda}^{2}(\Delta[0])$ defined by $w\left(s_{0}^{2}(0)\right)=1$. The map $\rho_{G}: A_{G}^{*}([0]) \longrightarrow S_{\lambda}^{*}(\Delta[0])$ is uniquely determined. In order to show that it is an isomorphism, because of the multiplicative structure, it is enough to show that $\rho_{G}\left(u_{0}\right) \neq 0$. Calculating (i.e., going through the details of $[\mathrm{B}, \mathrm{G}]$, Lemma 2.3 and Proposition 2.4, and not forgetting to average the homotopies) one finds that $\rho_{G}\left(u_{0}\right)=-\frac{1}{2} w$. 


\section{APPENDIX}

Proof of Lemma 2.3. The case where $k=0$ is clear; and so we proceed by induction, assuming that $k>0$ and that the result is proven up to $k-1$. Without loss of generality we can write the relation in the form

$$
s_{i_{1}} \ldots s_{i_{k}} x=t_{n+k}^{m} s_{j_{1}} \ldots s_{j_{k}} x
$$

where $i_{1}>\cdots>i_{k}$.

Now in $\Delta C^{o p}$ one has that (for $0 \leq m \leq n+k$ )

$$
t_{n+k}^{m} s_{j}= \begin{cases}s_{j+m} t_{n+k-1}^{m} & \text { if } j+m<n+k, \\ s_{j+m-(n+k+1)} t_{n+k-1}^{m-1} & \text { if } j+m>n+k, \\ t_{n+k} s_{n+k-1} t_{n+k-1}^{m-1} & \text { if } j+m=n+k .\end{cases}
$$

Case 1. $j_{1}+m \neq n+k$. So we have a relation of the form

$$
s_{i_{1}} \ldots s_{i_{k}} x=s_{j^{\prime}} t_{n+k-1}^{m^{\prime}} s_{j_{2}} \ldots s_{j_{k}} x .
$$

If $j^{\prime}>i_{1}$, applying $d_{j^{\prime}+1}$ gives $s_{i_{1}} \ldots s_{i_{k}} d_{j^{\prime}+1-k} x=t_{n+k-1}^{m^{\prime}} s_{j_{2}} \ldots s_{j_{k}} x$. Whence $x=d_{j_{k}} \ldots d_{j_{2}} t_{n+k-1}^{-m^{\prime}} s_{i_{1}} \ldots s_{i_{k}} d_{j^{\prime}+1-k} x$. Now writing the operator on the right in standard form ( $T S D$, where $T$ is a cyclic group operator, $S$ is a sequence of degeneracies and $D$ is a sequence of boundaries) shows that some $t_{n}^{s} x$ is degenerate-a contradiction.

If $j^{\prime} \leq i_{1}$, first suppose that no index $i_{t}=j^{\prime}$ or $j^{\prime}-1$. Then applying $d_{j^{\prime}}$ gives a contradiction as before. If some $i_{t}=j^{\prime}$, then applying $d_{j^{\prime}}$, the inductive assumption and $s_{j^{\prime}}$ gives the result. If no $i_{t}=j^{\prime}$ but some $i_{t}=j^{\prime}-1$, then applying $d_{j^{\prime}}$, the inductive assumption and $s_{j^{\prime}}$ gives $s_{i_{1}} \ldots s_{j^{\prime}} \ldots s_{i_{k}} z=$ $t_{n+k}^{m} s_{j_{1}} \ldots s_{j_{k}} z$ and $s_{i_{1}} \ldots s_{j^{\prime}} \ldots s_{i_{k}} x=s_{i_{1}} \ldots s_{j^{\prime}-1} \ldots s_{i_{k}} x$. Applying $d_{i_{1}}$, the inductive assumption and $s_{i_{1}}$ to the latter gives the result.

Case 2. $j_{1}+m=n+k$. First suppose that $k \geq 2$. If $j_{1} \leq j_{2}$, then $s_{j_{1}} s_{j_{2}}=$ $s_{j_{2}+1} s_{j_{1}}$, and we are back in Case 1. If $j_{1}>j_{2}$, then $s_{j_{1}} s_{j_{2}}=s_{j_{2}} s_{j_{1}-1}$, and again we are back in Case 1 .

Thus we are left with the case where $k=1$ and $j_{1}+m=n+1$. The relation is $s_{i_{1}} x=t_{n+1}^{m} s_{j_{1}} x=t_{n+1} s_{n} t_{n}^{m-1} x$. Applying $d_{0}$ gives a contradiction of the non-degeneracy unless $i_{1}=0$.

So now we are left with $s_{0} x=t_{n+1} s_{n} t_{n}^{m-1} x$. Applying $d_{0}$ gives $x=t_{n}^{m-1} x$; and so $s_{0} x=t_{n+1} s_{n} x$. Now applying $d_{1}$ gives a contradiction of the nondegeneracy unless $n=0$. But, if $n=0$, we have $s_{0} x=t_{1} s_{0} x$, and hence $x \in Y_{0}^{f}$ - a contradiction.

Proof of Corollary 2.4. Suppose that

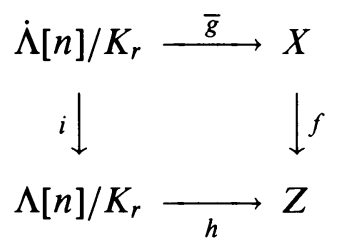


is a commutative diagram of cyclic sets. Define $\varphi: X \cup\langle x\rangle \longrightarrow Z$ by $\varphi \mid X=f$ and $\varphi(x)=h\left(\left[1, l_{n}\right]\right)$. We must check that $\varphi$ is extendable as a map of cyclic sets. (Clearly $\varphi$ is unique.)

Let $T S D$ be a cyclic operator, where $T$ is a cyclic group operator, $S$ is a sequence of degeneracies and $D$ is a sequence of boundaries. If $D$ is non-trivial, then $T S D x \in X$; and so $\varphi(T S D x)=f(T S D x)=T S f(D x)=$ $\operatorname{TSf} \bar{g}\left(D\left(\left[1, l_{n}\right]\right)\right)=\operatorname{TShiD}\left(\left[1, l_{n}\right]\right)=\operatorname{TSDh}\left(\left[1, l_{n}\right]\right)=\operatorname{TSD} \varphi(x)$.

If $D$ is trivial, and $T S x=T^{\prime} S^{\prime} D^{\prime} x$, then $D^{\prime}$ is trivial since $T S$ has a left inverse and $x \notin X$. So $T S x=T^{\prime} S^{\prime} x$. Hence, by Lemma 2.3, $T S \varphi(x)=$ $T^{\prime} S^{\prime} \varphi(x)$. Thus $\varphi$ extends as a map of cyclic sets.

\section{REFERENCES}

[A, P] C. Allday and V. Puppe, Cohomological methods in transformation groups, Cambridge Studies in Advanced Mathematics 32, Cambridge Univ. Press, Cambridge, 1993.

[B, H, M] M. Bökstedt, W.-C. Hsiang and I. Madsen, The cyclotomic trace and algebraic $K$-theory of spaces, Invent. Math. 111 (1993), 465-539.

[B, G] A. K. Bousfield and V. K. A. M. Gugenheim, On PL de Rham Theory and rational homotopy type, Mem. Amer. Math. Soc. 179 (1976).

[D, H, K] W. G. Dwyer, M. J. Hopkins and D. M. Kan, The homotopy theory of cyclic sets, Trans. Amer. Math. Soc. 291 (1985), 281-289.

[H] S. Halperin, Lectures on minimal models, Mém. Soc. Math, France (N.S.), No. 9-10 (1983).

[J] J. D. S. Jones, Cyclic homology and equivariant homology, Invent. Math. 87 (1987), 403-423.

[L] J.-L. Loday, Cyclic homology, Grundlehren. Math. Wiss. 301, Springer-Verlag, Berlin, Heidelberg, 1992.

[M] J. P. May, Simplicial objects in algebraic topology, Van Nostrand Math. Studies, no. 11, Van Nostrand, Princeton, NJ, 1967.

[Sp] J. Spaliński, Strong homotopy theory of cyclic sets, J. Pure Appl. Algebra 99 (1995), 35-52.

[S] D. Sullivan, Infinitesimal computations in topology, Publ. Math. I.H.E.S. 47 (1977), 269-332.

Department of Mathematics, University of Hawail, Honolulu, Hawail 96822

E-mail address: allday@uhunix.uhcc.hawaii.edu or chris@math.hawaii.edu 